\title{
Bringing Light - Addressing the Sexual and Reproductive Health and Rights (SRHR) of People Living with Physical Disabilities (PWDPs)
}

\author{
Junaid-Ur-Rehman Siddiqui*, Naureen Lalani, Sheena Hadi, Aisha Ijaz and Hira Mustafa Khan \\ Aahung, Sasi Homes, G-18/6, Block 8, Clifton, Karachi, Pakistan \\ ${ }^{\star}$ Corresponding author: Junaid-ur-Rehman Siddiqui, Aahung, Sasi Homes, G-18/6, Block 8, Clifton, Karachi, Pakistan
}

Received: March 08, 2021; Accepted: March 15, 2021; Published: March 24, 2021

\begin{abstract}
In Pakistan, there is a 'culture of silence' around disability and Sexual and Reproductive Health (SRH) rights. Therefore, SRH needs and rights of people living with disabilities remain unaddressed because of prevailing cultural norm and traditions that stigmatize sexuality of People Living with Disability (PWD) and prevent them from claiming their sexual rights and taking control of their reproductive lives. Furthermore, people with disabilities are unable to access quality and tailored SRHR information and services.

The aim of this project was to build the capacity of People Living with Physical Disabilities (PWPD) on their Sexual and Reproductive Health and Rights (SRHR). The project was also aimed at increasing awareness among the general public on the SRHR needs of PWPDs as they are generally considered asexual, hence not needing access to SRHR information and services. To achieve this, Aahung planned to build capacity of the caregivers, and trainers who work with/for people living with disabilities and develop user-friendly resource material to aid them to teach people living with physical disability about their SRH needs and rights which is an innovation within itself. The approaches discussed here, however, apply broadly to all aspects of health programming for people with physical disabilities. Aahung envisioned transforming these trainers into advocates for SRH needs and rights of PWPDs in their respective workspaces and/or communities and integrate the newly-designed SRHR-related resource material into their activities.
\end{abstract}

\section{Introduction}

Sexual and reproductive health and rights (SRHR) fulfillment is essential to an individual's overall wellbeing and prosperity, but collectively, it plays a greater and much more crucial role in the development of a nation [1]. Progressive SRHR attitudes and behaviours in general population are critical for sustainable human development, especially in a developing country like Pakistan [2,3]. SRHR encompasses comprehensive information and services directed toward tackling gender biases, rights violation, sexual and genderbased violence, and concerns regarding adolescence, puberty, and sex and sexuality; it also ensures provision of reliable information and services around family planning, contraception, and post-abortion care in order to promote safe and healthy SRHR behaviours $[1,4]$. However, strong stigma is associated with these topics in the country, resulting in none to limited conversation and consequent low general knowledge, misinformation, negative perceptions and attitudes, and unhealthy practices around SRH.

People living with physical disabilities are further distanced from SRH resources owing to the common misconception that they are not sexual beings or sexually active [5]. Researchers have found that People with Physical Disabilities (PWPDs) are usually stereotyped as asexual, which can lead to significant sexual and reproductive disparities when compared with the population living without any disability
[6,7]. Several other studies further highlight the different kinds of neglect and discrimination faced by PWPDs. A research found that they are less likely to receive higher education and are usually socially isolated. Impaired interpersonal relations and social communication can lead to reduced self-esteem and confidence in physically disabled people, which can prevent them from claiming their sexuality and sexual concept and from accessing desired Sexual and Reproductive (SRH) services [8]. The lack of self-esteem in PWPDs has also been vastly reported to result in significantly low sexual esteem, low sexual satisfaction, and high sexual distress [7,9].

According to Pakistan Bureau of Statistics, there are 3.3 million people with disabilities in the country, which is $1.6 \%$ of the total population [10]. However, the country's data on PWPDs is insufficient and unreliable, major reasons being non-cooperation of respondents and inconsistency in the definition of 'disability'. Currently, there is no widely accepted definition of 'people with disabilities' in Pakistan's national policies [11]. In Pakistan, there is a 'culture of silence' around the needs of people that are living with physical disabilities, especially their sexual and reproductive health, needs, and rights. The prevalence of discouraging cultural norms and traditions that stigmatize sexuality of people with disabilities leaves an essential component of their lives largely unaddressed and prevents them from claiming their sexual health and rights and taking control of their reproductive lives. Furthermore, PWPDs face a greater challenge when they are unable to access quality 
SRHR information and services that are exclusively tailored to their needs. The success or usefulness of any programme relies largely on the shared needs and wishes of the beneficiaries and key stakeholders. In Pakistan, however, no effort has been made to scientifically assess genuine SRH needs and problems of the PWPDs [11].

It is imperative to realize that the main reason for the disconnect between PWPDs and their access to SRHR is not the disability itself, but the prevailing assumptions regarding their needs among service providers, communities, and the policy makers [12]. The ignorance that pervades our society with regards to sexuality and disability renders the SRH needs and rights of PWPDs absent [13]. The misrepresentation and social exclusion combined with lack of access to the necessary resources, results in increased vulnerability of the physically disabled people to Sexually Transmitted Infections (STIs); they are also more likely to experience physical, sexual, and emotional violence and mental health issues $[14,15]$. To overcome the breach in equal provision of SRH information and services, it is recommended to increase the competency and capacity of organizations and care/ service providers working for PWPDs, as well as the PWPDs themselves. Additionally, there is a strong need to conduct researches to identify, explore, and effectually employ evidence-based solutions [12]. Aahung designed a comprehensive module to build capacity of the caregivers and trainers working with/for PWPDs, to inform their attitudes and perceptions around the various themes of SRH with physical disability as a cross-cutting theme. Aahung envisioned transforming these trainers into advocates for SRH needs and rights of PWPDs in their respective workspaces and/or communities and integrate the newly-designed SRHR-related resource material into their activities.

\section{Methods}

\section{Intervention}

Aahung established partnerships with four different organizations working for the welfare and wellbeing of PWPDs, namely NOWPDP, Center for Inclusive Care, BINAE foundation and Connect Hear. From these partner organizations, 19 participants were identified to be trained as Master Trainers on the SRHR needs of PWPDs. Participants included trainers working in relevant organizations, care givers, and PWPDs.

Aahung developed user-friendly resource material and training modules for capacity building after consulting with organizations working for the people living with physical disabilities; data and results from Aahung's previous programmers were also taken into consideration to strengthen the training module. For people with hearing impairments, animated videos were dubbed into sign language on SRHR issues including gender, puberty, sexual abuse, early age marriage, family planning, and abortion. The content was translated into braille, by experts, for people with visual impairments and videos were dubbed into sign language for people with hearing impairments. The translations were verified by the receivers themselves; when introduced to the material, individuals with seeing or hearing disabilities were able to understand the content. Additionally, interpreters and training facilitators were also made part of the training. The facilitators were identified and employed on the basis of their expertise in SRH. Training facilitators also included PWPDs to improve communication with participants. Trained participants reached out to approximately 200 people, sensitizing them on the SRHR needs of PWPDs.

\section{Study Design and Setting}

Training was held in Karachi in June, 2018. It spanned over three days, covering topics including Social Determinants of Health, Value Clarification and Attitudinal Transformation (VCAT), Gender, Sex and Sexuality, Sexual and Reproductive Health and Rights, Puberty Changes, and Family Planning. The objective of training was to increase comfort of the participants for addressing issues related to SRHR and physical disability, to enhance knowledge of the participants on SRHR and physical disability, and to enhance skills of the participants to discuss issues related to SRHR and physical disability with other people.

\section{Data Collection and Management}

To assess the effectiveness of the intervention, participants' knowledge and beliefs were assessed through a pre- and post-test questionnaire. The questionnaire was developed in English and was translated to Urdu and Pakistan Sign Language for the hearingimpaired participants. Qualitative feedback was gathered as well from the participants to understand the experiential aspect. Questions focused on participants' understanding of puberty, gender, sexual and reproductive health rights, and family planning.

\section{Ethical Considerations}

All participants were given detailed information, verbally and in sign language, about the training including; objectives, anticipated benefits, expectations from the participants, the time that the training session will take, the pre- and post- tests, the fact that they may choose not to participate or to withdraw from it at any time, without reprisal. Verbal and sign language consent was taken from all participants. Measures were taken to ensure confidentiality and anonymity of the information provided by the participants.

\section{Results}

There was a substantial increase in the knowledge and attitude of participants post-training. $82 \%$ caregivers reported that they had insufficient information, and, therefore, felt unprepared to appropriately address the SRHR issues of the people living with physical disabilities. However, upon being reinforced with accurate knowledge and appropriate language after the training, $91 \%$ of the participants felt confident enough to interact of the matter with PWPDs and other relevant stakeholders.

At pre-test, $55 \%$ participants were able to identify the differences between sex and gender, however, at post-test, all participants were able to identify the differences.

"I thought "Sex" and "Gender" are the same thing before coming to this training, but now I understand that they are conceptually distinct." - Training Participant 
Knowledge related to girl's puberty was $0 \%$ before the training and increased to $64 \%$ afterwards. Similarly, knowledge about boy's puberty was $1 \%$ and increased to $73 \%$ after the training.

"I did not have any idea about pubertal changes when I was in my puberty age. This session should be attended by every child who is entering their pubertal age, so that they don't face difficulty in understanding the physical and emotional transitions." - Training Participant

The training was able to debunk misconceptions around sexual health as well. The greatest change in perception was seen for the myth stating ejaculation is unhealthy and causes weakness. $54 \%$ of the participants believed that ejaculation causes weakness and infertility whereas after the training no participant agreed with this.

Overall, the trainings were well-received by the participants.

"SRHR is not something that is spoken about in the disabled community (hearing and visually impaired), therefore this training was an opportunity to learn something new and share with others." Training Participant

The Master Trainers produced as the result of Aahung's training directly reached out to a total 200 PWPDs. The new trainees were from within the master trainers' organizations, communities, and social networks, and comprised PWPDs as well as caregivers. Aahung monitored and supported three of these trickledown trainings conducted by CIC, Connect Hear and BINAE foundation.

A telephonic follow-up was also conducted with the master trainers from the first training. They reported that the module introduced to them had immensely helped them in trickling down the message with other people living with physical disability and those providing services to them.

\section{Discussion}

Participants felt that the videos and activities were an effective way to understand and apply the knowledge learned, however, the interactive activities were more useful than those involving writing, because for those with physical disabilities, discussions were easier and a more effective way of communicating than writing their responses. Furthermore, interactive and user-friendly resource materials designed for specific disabilities played a vital role in sensitizing PWPDs on SRHR subjects generally considered to be taboos. Given that PWPDs are reluctant to reach out to service providers for fear of being mocked and judged, these interactive materials are an effective strategy to address their SRHR-related myths and misconceptions.

The intervention served as a pilot unveiling potential for scaling up the SRHR program with PWPDs on regional and national levels. Introducing educational material around SRHR in the forms of braille books and AV tools among people with various disabilities, allowed the researchers to learn regarding the extent of ease or difficulty of its receptivity by the target audience, as well as the measure of impact the specially designed module was able to produce. These findings will serve to further inform and update the program, which is then planned to be integrated into schools and other organizations and/or departments working for the benefit of PWPDs. Organizations that will be approached and involved for the integration of SRHR education for PWPDs of all ages and backgrounds, on state level, include, People with Disabilities Network Department of Health, Department of Education (especially the wing working on Life Skills Based Education (LSBE)), Pakistan Associations of the Blind (PAB), and Pakistan Association of the Deaf (PAD). Welfare organizations that provide vocational training for PWPDs will also be approached with the proposal to include SRHR module among their other forms of training. In Punjab, a civil society organization is working on a similar cause, but the organization primarily works around LSBE with younger individuals with disabilities, which can provide the window for Aahung to propose a similar program for their audience's SRHR needs.

Apart from involving public and private organizations in this cause by collaborating or partnering with them on state and local levels, an essential purpose for piloting the SRHR program with PWPDs was to study how eventually it could be mainstreamed into Aahung's regular LSBE and SRHM programmes. In the future, all Aahung trainings done by Aahung with students, parents, teachers, and healthcare providers among others are planned to have a segment on building awareness and destigmatizing the SRHR service and knowledge needs of PWPDs.

\section{Limitations}

Catering to people with different physical disabilities within one training was a challenge, since different disabilities require different and unique means to be addressed. During the trickle-down trainings, however, the MTs were able to overcome this challenge by conducting separate trainings for people with different physical disabilities. Furthermore, since there isn't any precedent for a similar programme in Pakistan and the exploratory nature of this intervention, a rigorous evaluation could not be done. Therefore, future studies should explore methodologies for evaluation with PWDs while incorporating different types of data collection methodologies suited to each disability.

\section{References}

1. Temmerman M, Khosla R, Say L (2014) Sexual and reproductive health and rights: A globaldevelopment, health, and human rights priority. The Lancet 384: e30-e31. [crossref]

2. Adinma J, Adinma E (2011) Impact of Reproductive Health on Socio-economic Development: A Case Study of Nigeria. African Journal of Reproductive Health 15: 7-12. [crossref]

3. Pillai VK, Maleku A (2015) Reproductive Health and Social Development in Developing Countries:Changes and Interrelationships. British Journal of Social Work 45: 842-860.

4. Braeken D, Rondinelli I (2012) Sexual and reproductive health needs of young people: Matching needswith systems. International Journal of Gynecology \& Obstetrics 119: S60-S63. [crossref]

5. DeBeaudrap P, Mouté C, Pasquier E, Mac-Seing M, Mukangwije P, et al. (2019) Disability and Access to Sexual and Reproductive Health Services in Cameroon: A Mediation Analysis of the Role of Socioeconomic Factors. International Journal of Environmental Research and Public Health 16: 417. [crossref]

6. Chance RS (2002) To Love and be Loved: Sexuality and People with Physical Disabilities. Journal of Psychology and Theology 30: 195-208.

7. Rowen TS, Stein S, Tepper M (2015) Sexual Health Care for People with Physical Disabilities. The Journal of Sexual Medicine 12: 584-589. [crossref]

8. Aron L, Loprest P (2012) Disability and the Education System. The Future of Children 22: 97-122. [crossref] 
Junaid-Ur-Rehman Siddiqui (2021) Bringing Light - Addressing the Sexual and Reproductive Health and Rights (SRHR) of People Living with Physical Disabilities (PWDPs)

9. McCabe MP, Taleporos G (2003) Sexual Esteem, Sexual Satisfaction, and Sexual Behavior among People with Physical Disability. Archives of Sexual Behavior 32: 359369. [crossref]

10. Pakistan Bureau of Statistics (2017) 6th Population and Housing Census-2017 (Population and HousingCensus). Government of Pakistan.

11. Ahmed M, Khan AB, Nasem F (2011) Policies for Special Persons in Pakistan Analysis of PolicyImplementation. Berkeley Journal of Social Sciences 1.

12. Oosterhoff P (2018) Sexual and Reproductive Health Rights of Persons With Disabilities (IDS Disability Briefing). Institute of Development Studies.
13. Addlakha R, Price J, Heidari S (2017) Disability and sexuality: Claiming sexual and reproductive rights. Reproductive Health Matters 25: 4-9. [crossref]

14. Aragão J da S, França ISX de, Coura AS, Medeiros CCM, Enders BC (2016) Vulnerability associated with sexually transmitted infections in physically disabled people. Ciência \& Saúde Coletiva 21: 3143-3152. [crossref]

15. Krnjacki L, Emerson E, Llewellyn G, Kavanagh AM (2016) Prevalence and risk of violence against people with and without disabilities: Findings from an Australian population-based study. Australian andNew Zealand Journal of Public Health 40: 1621. [crossref] 